Article

\title{
Development of Antioxidant-Loaded Nanoliposomes Employing Lecithins with Different Purity Grades
}

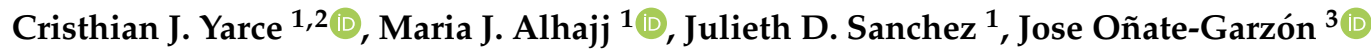 \\ and Constain H. Salamanca $1,2, *$ (1)
}

1 Laboratorio de Diseño y Formulación de Productos Químicos y Derivados, Departamento de Ciencias Farmacéuticas, Facultad de Ciencias Naturales, Universidad ICESI, Calle 18 No. 122-135, 760035 Cali, Colombia; cjyarce@icesi.edu.co (C.J.Y.); mariajoalhajj@hotmail.com (M.J.A.); julieths28@outlook.com (J.D.S.)

2 Centro de Ingredientes Naturales Especializados y Biotecnológicos-CINEB, Facultad de Ciencias Naturales, Universidad ICESI, Calle 18 No. 122-135, 760035 Cali, Colombia

3 Facultad de Ciencias Básicas, Programa de Microbiología, Universidad Santiago de Cali, Calle 5 No. 62-00, 760035 Cali, Colombia; jose.onate00@usc.edu.co

* Correspondence: chsm70@gmail.com

Academic Editors: Severina Pacifico and Simona Piccolella

Received: 20 October 2020; Accepted: 11 November 2020; Published: 16 November 2020

\begin{abstract}
This work focused on comparing the ability of lecithins with two purity grades regarding their performance in the development of nanoliposomes, as well as their ability to contain and release polar (trans-aconitic acid) and non-polar (quercetin) antioxidant compounds. First, the chemical characterization of both lecithins was carried out through infrared spectroscopy (FTIR), electrospray ionization mass spectrometry (ESI/MS), and modulated differential scanning calorimetry (mDSC). Second, nanoliposomes were prepared by the ethanol injection method and characterized by means of particle size, polydispersity, and zeta potential measurements. Third, the encapsulation efficiency and in vitro release profiles of antioxidants were evaluated. Finally, the antioxidant effect of quercetin and trans aconitic acid in the presence and absence of nanoliposomes was assessed through the oxygen radical absorbance capacity (ORAC) assay. The results showed that, although there are differences in the chemical composition between the two lecithins, these allow the development of nanoliposomes with very similar physicochemical features. Likewise, nanoliposomes elaborated with low purity grade lecithins favored the encapsulation and release of trans-aconitic acid (TAA), while the nanoliposomes made with high purity lecithins favored the encapsulation of quercetin (QCT) and modified its release. Regarding the antioxidant effect, the vehiculization of TAA and QCT in nanoliposomes led to an increase in the antioxidant capability, where QCT showed a sustained effect over time and TAA exhibited a rapidly decaying effect. Likewise, liposomal systems were also found to have a slight antioxidant effect.
\end{abstract}

Keywords: antioxidant effect; lectins; nanoliposomes; purity grade; quercetin; trans-aconitic acid

\section{Introduction}

Lecithins are a type of raw material widely used by different sectors, such as pharmaceutical, food, and cosmetic sectors, where they are employed for different purposes due to their great versatility [1]. Some of these uses correspond to their ability to act as emulsifiers, as well as humectants and suspension stabilizers [2]. However, their most important application lies in their ability to form nanoliposomes, which represent a very special type of self-assembling system that is very useful as a nanometric formulation vehicle for multiple compounds of interest in the previously mentioned sectors [3,4]. 
Lecithins are complex raw materials because they consist of a mixture of various types of phospholipids, where phosphatidylcholine (PC) is usually the main component [5]. However, they may also contain other types of phospholipids, such as phosphatidylethanolamines (PE), phosphatidylserines (PS), phosphatidylinositols (PI), phosphatidylglycerols (PG), and glyphospholipids (GLP), as well as other components, such as phosphatidic acid (PA) and cholesterol [6]. Therefore, depending on the obtention source (vegetable or animal), the type of extraction, and the purification methods, lecithins can be composed of different types and amounts of phospholipids and thus have a wide diversity of references with multiple purity grades and commercial prizes [7]. This diversity of references can, in some cases, lead to confusion or a lack of criteria for choosing the correct lecithin. In the case of the pharmaceutical sector, this choice is easier to make due to the intrinsic features of such products and their regulatory affairs, in which it is practically mandatory to use raw materials with the highest possible purity grade. In contrast, this situation is different in other sectors, such as foodstuff and cosmetic sectors, where lower purity grade lecithins can be used with less restrictions. Therefore, the question presented in this study is as follows: Can a low purity lecithin develop nanoliposomes, just like a high purity lecithin? To address this question, the work focused on (i) chemically characterizing two types of soybean lectins with different purity grades and commercial value; (ii) the development and characterization of nanoliposomes loaded with two model antioxidants, corresponding to quercetin [8] and trans-aconitic acid [9]; and (iii) determining the encapsulation efficiency and in vitro release profiles of antioxidant compounds from nanoliposomal vehicles.

\section{Results and Discussion}

\subsection{Lecithin Characterization}

Firstly, it is important to highlight that for the comparison of the purity grade to be consistent, it is necessary that lecithins come from the same origin and therefore have a similar phospholipid composition. If this was not the case, the results would be meaningless and may not be reproduced. Considering this, the results discussed as the conclusions issued in this manuscript correspond to lecithins from a similar plant source (soybean). In the case of low purity lecithin, this was found to be a brown fluid with a sticky consistency. In contrast, the high purity lecithin was shown to be a yellowish granular waxy solid that could be easily handled. Such physical appearance characteristics are a very important factor to consider in foodstuff raw materials, since, depending on their consistency, various stages of product development, such as dispensing, weighing, and post-production cleaning, may be easier to carry out. Therefore, low purity lecithin has a disadvantage compared to high purity lecithin, which is easier to handle. Additionally, the color of the raw materials is another aspect to be considered, since the appearance of these plays an important role in the sensory characteristics of the product to be developed, where dark colors are more difficult to mask, which may be another disadvantage of low purity lecithin versus high purity lecithin. Consequently, the physical appearance of these lecithins is largely determined by their own chemical composition. In this way, and according to the respective technical data sheets, low purity lecithin has a distribution of phospholipids of around $90 \%$, where $\sim 50 \%$ is phosphatidylcholine, $\sim 30 \%$ are inositol phosphatides, and $\sim 10 \%$ is phosphatidylethanolamine; the remaining $10 \%$ corresponds to several impurities [5,10]. In contrast, high purity lecithin consists of $97 \%$ phospholipids, where $\sim 92 \%$ is phosphatidylcholine, $\sim 3 \%$ is lysophosphatidylcholine, and $\sim 2 \%$ are other phosphatides, with the remainder also corresponding to compounds other than phospholipids [5].

\subsubsection{FTIR Characterization}

Figure 1A shows the FTIR spectra for low and high purity lecithins and also indicates the chemical structures of the two phospholipids that, according to the literature, are present in a greater proportion in lecithins [5]. In the case of Lp-SBL, signals were found at 3384, 3010, 2924, and $2854 \mathrm{~cm}^{-1}$. These signals correspond to the stretching vibrations of the $\mathrm{OH}$ groups from (i) the carboxylic acid 
function of the fatty acids and the saccharides present in glyphospholipids; (ii) the amino group present in some phospholipids, such as phosphatidylcholine; and (iii) the symmetric and asymmetric tension vibrations of the $\mathrm{CH}_{2}$ groups present in fatty acids. In the case of Hp-SBL, the signals were observed at 3334, 3009, 2924, and $2854 \mathrm{~cm}^{-1}$. Moreover, the $\mathrm{OH}$ band amplitude and intensity suggest that low purity lecithin has a higher content of saccharides. On the other hand, the bands of $1744 \mathrm{~cm}^{-1}$ for Lp-SBL and $1739 \mathrm{~cm}^{-1}$ for Hp-SBL correspond to the stretching vibration of the carbonyl bond $(\mathrm{C}=\mathrm{O})$. However, the signal shifting suggests that the substitution for carbonyl is different in each raw material, which is due to differences in the length and proportion of substituent fatty acids in phospholipids. Likewise, the multiple bands that appear for both raw materials between 1238 and $1064 \mathrm{~cm}^{-1}$ correspond to the phosphate groups in the phospholipid chains. Finally, the wide shapes of the signals presented between 1064 and $1165 \mathrm{~cm}^{-1}$ in low purity lecithin are representative of the asymmetric tensions of $\mathrm{OH}$ groups present in sugars, which is consistent with a higher amount of saccharide molecules in that lecithin.

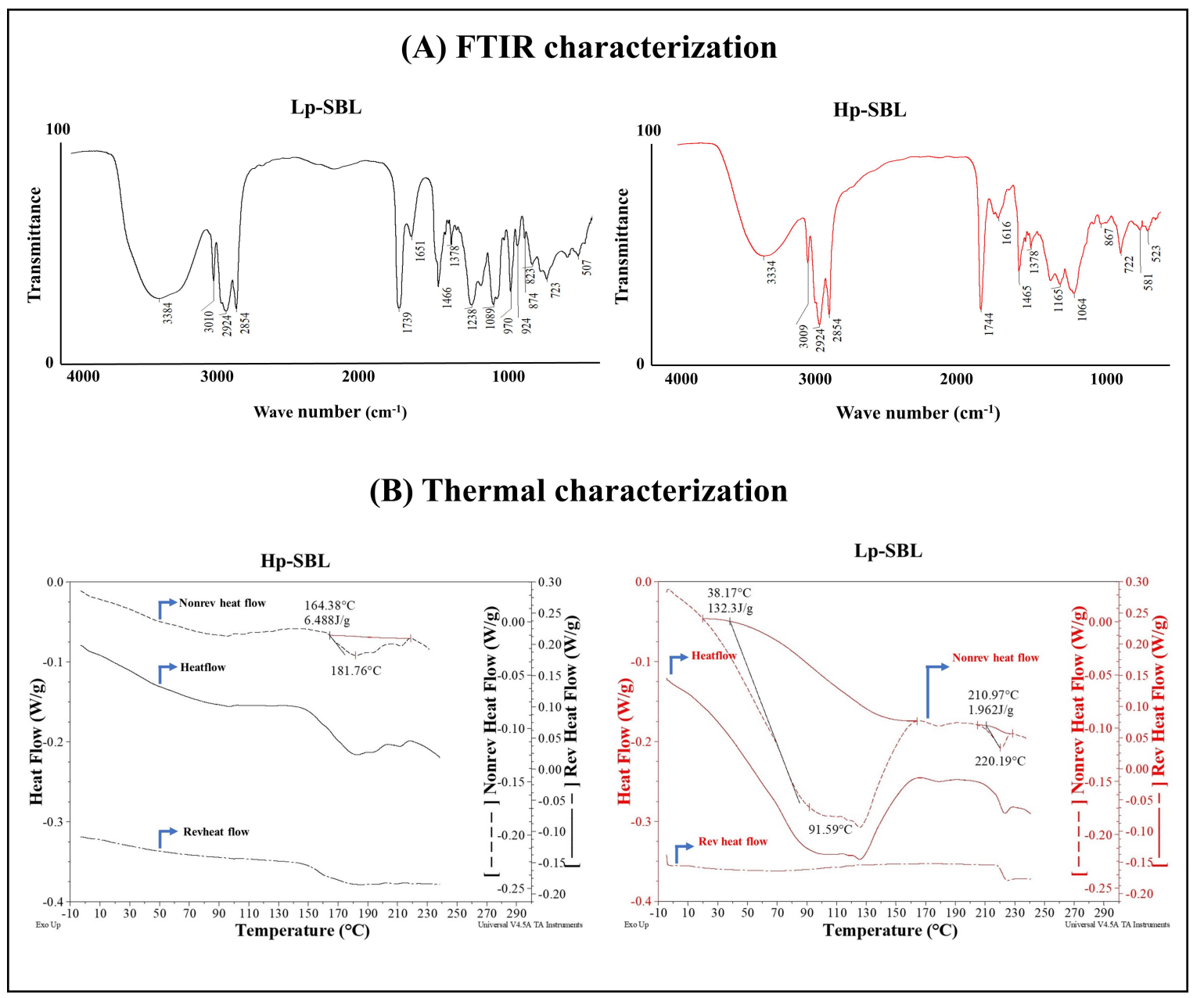

Figure 1. (A) Infrared spectroscopy (FTIR) signals for low purity grade (Lp-SBL) and high purity grade (Hp-SBL) soybean lecithins. (B) Modulated differential scanning calorimetry (mDSC) signals for low purity grade (Lp-SBL) and high purity grade (Hp-SBL) soybean lecithins. The solid line corresponds to the total heat flow, the short dashed line corresponds to the non-reversible heat flow, and the broken dashed line corresponds to the reversible heat flow.

\subsubsection{Thermal Analysis}

The results of the thermal characterization performed are shown in Figure 1B. In the case of low purity lecithin, it is possible to observe two thermal transitions. The first one had an onset at approximately $38.2{ }^{\circ} \mathrm{C}$, a transition peak at around $91.6^{\circ} \mathrm{C}$, and an enthalpy value of $132.3 \mathrm{~J} / \mathrm{g}$. 
This behavior can be observed in the total heat flow signal (solid line) and in the non-reversible heat flow signal (short dashed line), but not in the reversible heat flow signal (dashed line). This indicates that there is an endothermic-type transition caused by the kinetics of the molecules inside the material [11-13]. It leads to a reorganization and presents multiple rearrangements in their physical structure, suggesting a possible loss of volatile substances and water present in the Lp-SBL raw material. Consequently, according to the enthalpy of the transition, the energy expenditure required to remove volatile substances and water was relatively high, due to the contribution of the heat of water vaporization [12-17]. The DSC results for Lp-SBL are consistent with the mass spectra, where representative signs of mass loss due to the dehydration of saccharide-like molecules were observed.

On the other hand, in the case of high purity lecithin, the total heat flow signal presents an endothermic-type transition, with an onset at around $164.4^{\circ} \mathrm{C}$, a peak at $181.8^{\circ} \mathrm{C}$, and an enthalpy of $6.5 \mathrm{~J} / \mathrm{g}$. These signals are consistent for the reversible heat flux and the non-reversible heat flux. This shows that the high purity lecithin is a consistent material and that its composition is defined by compounds that operate around approximate temperature values. Furthermore, due to the relatively low enthalpy of the transition, it establishes the heat flow through the material being transmitted homogeneously, which requires a low energy expenditure for the fusion of phospholipids in Hp-SBL. Furthermore, for this raw material, there is no sign of water loss or signs due to the rearrangement of the material, as is the case for Lp-SBL. Finally, all these results are reliable in terms of the fluidity and physical appearance of the Lp-SBL, where it is necessary to remove the water and other volatile components prior to the fusion of the phospholipids, which is a transition that is displaced with an onset of $211.0^{\circ} \mathrm{C}$, with a peak at $220.2^{\circ} \mathrm{C}$ and an enthalpy of $1.96 \mathrm{~J} / \mathrm{g}$.

\subsubsection{Electrospray Ionization Mass Spectrometry (MS/ES $\left./ \mathrm{ES}^{+}\right)$}

Figure 2 shows the mass spectra recorded in negative $\left(\mathrm{ESI}^{-}\right)$and positive $\left(\mathrm{ESI}^{+}\right)$ion mode. The signals are discussed together for both Lp-SBL and Hp-SBL. In the case of the mass spectrum in $\mathrm{ESI}^{-}$negative ionization mode, (Figure 2A left), the $[\mathrm{M}+\mathrm{H}]$ ion is observed for phosphatidylcholine substituted with linoleic acid (C18:2) and palmitic acid (C16:0), at an $m / z$ ratio of 758 Da. Additionally, an ion with an $m / z$ of $833 \mathrm{Da}$, corresponding to phosphatidyl inositol, with substitutions of linoleic acid and oleic acid (C18:1), is observed. Conversely, in the case of the mass spectrum registered in positive $\mathrm{ESI}^{+}$mode (Figure 2A right), the presence of a molecular adduct of potassium for phosphatidylcholine, which is the ion $[\mathrm{M}+\mathrm{K}]$ with an $\mathrm{m} / \mathrm{z}$ ratio, can be found at $797 \mathrm{Da}$. In addition, the ion corresponding to the dehydrated form of [GLP] can be found at an $m / z$ ratio of $832 \mathrm{Da}$. It is important to highlight that, in the case of low purity lecithin, the abundance between $[\mathrm{M}+\mathrm{H}]$ and [GLP] is inverted regarding the abundance of these ions in high purity lecithin. Consequently, a greater abundance is observed for the phosphatidyl inositol signal than for the phosphatidylcholine signal, which is consistent with a higher presence of saccharides in the low purity lecithin.

On the other hand, Figure 2B shows the estimated fragmentation patterns in negative ionization mode for phosphatidylcholine and inositol glycophospholipid, respectively. It can be observed that the molecular ion $[\mathrm{M}+\mathrm{H}]$ for phosphatidylcholine suffers a loss of fraction (1) of $m / z 279 \mathrm{Da}$, which corresponds to the loss of the linoleic acid substituent of phosphatidylcholine, which leads to the generation of $479 \mathrm{Da} \mathrm{m} /$ fragment of $\mathrm{z}$ (3). In addition, a loss of $\mathrm{m} / \mathrm{z}$ of $255 \mathrm{Da}$, corresponding to the palmitic acid substituent and a fragment (4) of the $m / z$ ratio of $714 \mathrm{Da}$, is formed from the rupture of the nitrogen-bound methyl groups of the choline fraction. In Figure 3B, a water molecule from the phosphatidyl inositol [GLP] glycophospholipid is lost and forms a fragment with an $\mathrm{m} / \mathrm{z}$ ratio of 831 Da. These processes occur in both low purity lecithin and high purity lecithin. Furthermore, Figure $3 \mathrm{C}$ shows the fragmentation patterns for phosphatidylcholine and inositol glyphospholipids in positive ionization mode. In $\mathrm{ESI}^{+}$, a molecular adduct for phosphatidylcholine $[\mathrm{M}+\mathrm{K}]$ is presented, which suffers losses of the substituent fatty acids in the phospholipid (1) and the methyl groups bounded to nitrogen in the choline fraction (4). In the case of [GLP], a loss of the dehydrated inositol molecule (5) and the substituent fatty acids in the phospholipid (2) can be observed. Accordingly, 
it is important to highlight that the results suggest that the ions present in the low purity lecithin are also present in high purity lecithin. Therefore, phospholipids such as phosphatidylcholine and phosphatidyl inositol are present in both raw materials and it is thus possible to obtain the advantages of these compounds when using lecithins for a process of transformation or the subsequent preparation of a food or cosmetic product. However, it is estimated that the phosphatidylcholine:phosphatidyl inositol ratio is lower for Lp-SBL compared to Hp-SBL. Furthermore, it should be recognized that other phospholipids, such as phosphatidylethanolamine and phosphatidylserine, may also be present in the raw materials [10], but their signals were not observed, because they could be overlapped with the abundance of ions from other compounds in lecithins.

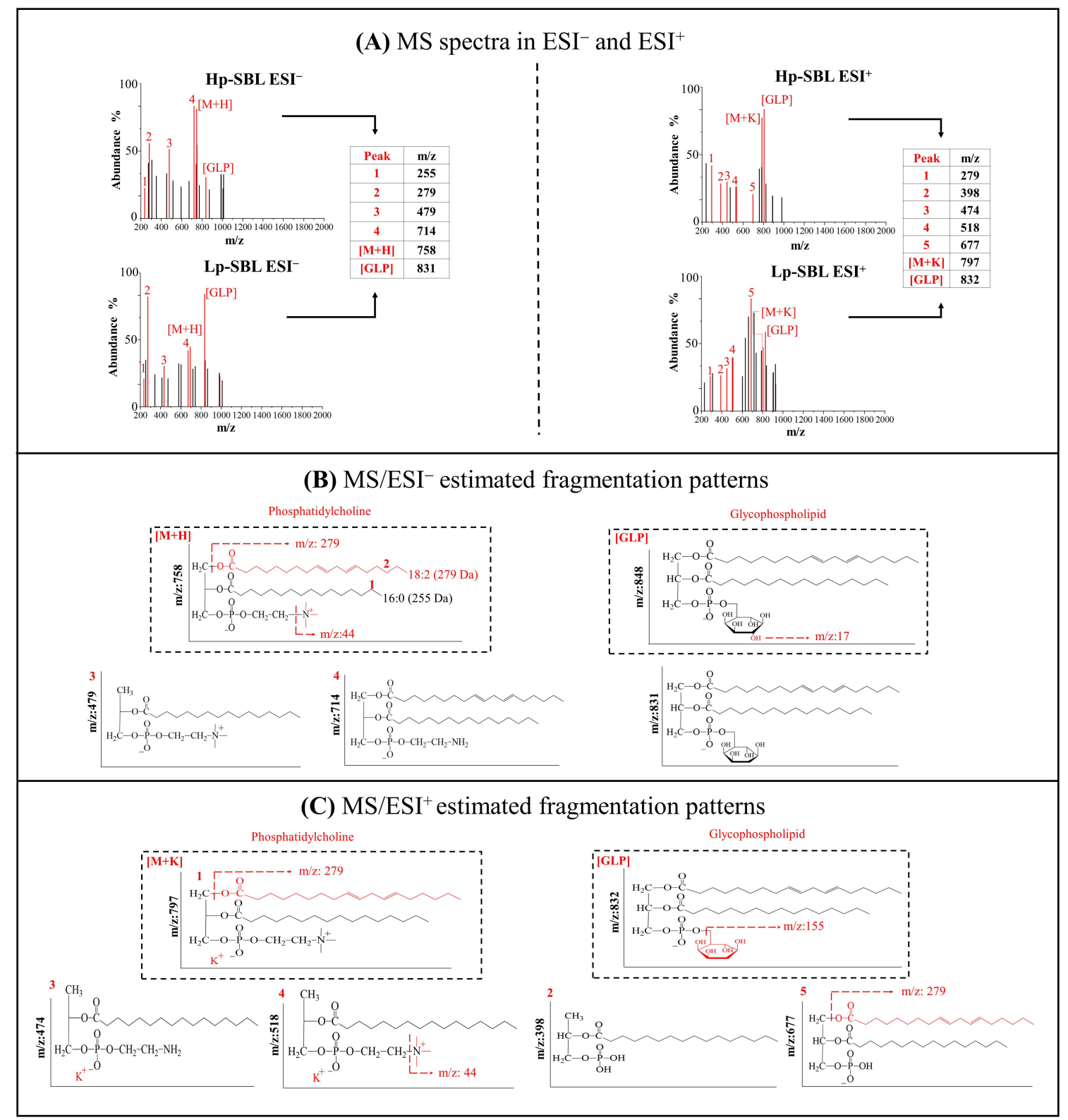

Figure 2. (A) Mass spectrometry (MS) spectra in total ion scanning mode for positive ion (ESI ${ }^{-}$) and negative ion $\left(\mathrm{ESI}^{+}\right)$modes for high purity lecithin (Hp-SBL) and low purity lecithin (Lp-SBL). (B) $\mathrm{MS} / \mathrm{ESI}^{-}$estimated fragmentation patterns. (C) $\mathrm{MS} / \mathrm{ESI}^{+}$estimated fragmentation patterns. 


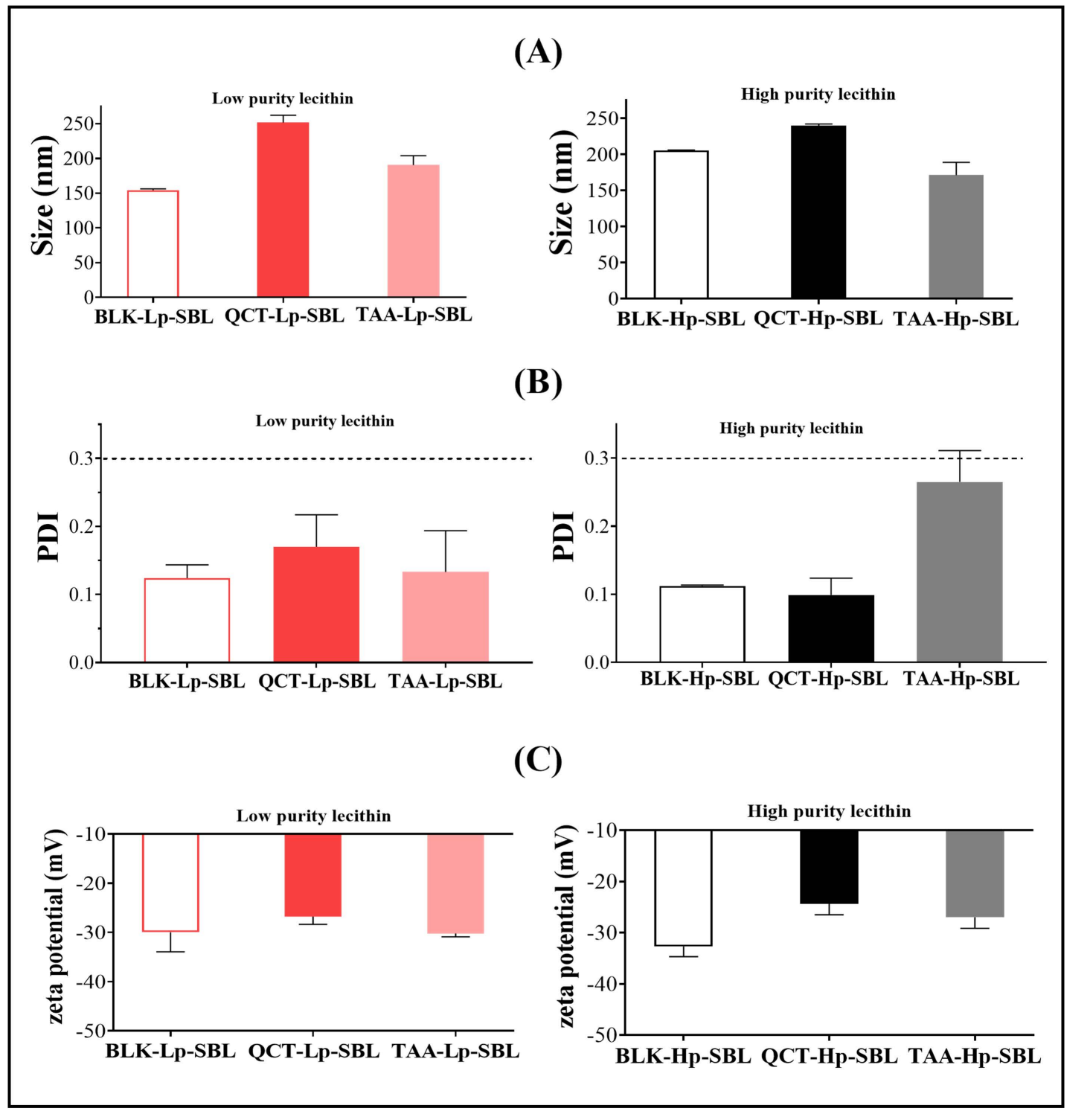

Figure 3. (A) Size, (B) polydispersity index (PDI), and (C) zeta potential for liposomes prepared with high purity lecithin (Hp-SBL) and low purity lecithin (Lp-SBL). BLK corresponds to unloaded nanoliposomes. Data are reported as the mean $\pm \mathrm{SD}$ and $n=3$.

\subsection{Development of Nanoliposomes}

\subsubsection{Physicochemical Characterization}

Figure 3 shows the results of the particle size, polydispersity index, and zeta potential for nanoliposomes prepared with low purity (Lp-SBL) and high purity (Hp-SBL) soybean lecithins. In the case of nanoliposomes prepared with Lp-SBL, the particle size of non-loaded nanoliposomes (BLK-Lp) was around $150 \mathrm{~nm}$, while the particle sizes of the nanoliposomes loaded with QCT (QCT-Lp) and TAA (TAA-Lp) were $\sim 250$ and $\sim 200 \mathrm{~nm}$, respectively. In contrast, for the nanoliposomes prepared with Hp-SBL, the size of non-loaded nanoliposomes (BLK-Hp) was around $200 \mathrm{~nm}$, while the sizes of the nanoliposomes loaded with QCT (QCT-Hp) and TAA (TAA-Hp) were 250 and 180 nm, respectively (Figure 3A). These differences in particle size can be explained considering the composition of phospholipids in both lectins and specifically, in those that consist of greater polar phospholipid amounts (inositol, saccharides, etc.), such as Lp-SBL, and that lead to a higher compaction of the aqueous liposomal core. Likewise, such a compaction effect can also be observed when the nanoliposomes are loaded with TAA, which is a very polar molecule [18-22]. On the other hand, it is important 
to highlight that the particle size reached in the liposomes prepared with both lecithins is on a nanometric scale (150-250 $\mathrm{nm}$ ), which favors the permeation process in many biological membranes and is, in fact, an interesting characteristic for the development of functional food products [23-25]. Regarding the polydispersity values (Figure 3B), it was found that, regardless of the lecithin used, it always tends to form nanoliposomes (loaded and unloaded) with a low particle size distribution (PDI < 0.3). This result is very interesting, since it suggests that both lecithins can be used to produce nanoliposomes of a regular size, which is essential to guaranteeing the uniformity of the content inside the nanoliposomes [4,26,27]. In relation to the zeta potential (Figure 3C), this parameter showed values of around $-30 \mathrm{mV}$ in all of the nanosystems obtained, regardless of the lecithin used. These negative zeta potential values can be explained considering that liposomes consist of a small portion of fatty acids (oleic acid, linoleic acid, etc.), which can be slightly ionized in the aqueous medium. In the same way, it can be considered that the autoprotolysis effect of water, where some hydroxy anions are generated and located in the liposome-aqueous medium interface, leads to an increase in the negative zeta potential [28]. Therefore, this result suggests that nanoliposomes could have an adequate physical stability, because of electrostatic repulsion that prevent interparticle aggregation [29-31]. Furthermore, the systems loaded with TAA lead to more negative zeta potential values. This result can be explained by considering that TAA is a polycarboxylic acid (carboxylic acid-carboxylate), which can also be adsorbed in the interfacial zone (a fraction), increasing the anionic charge in this zone and therefore, the zeta potential.

\subsubsection{Encapsulation and In Vitro Release of Antioxidant Compounds}

Figure $4 \mathrm{~A}$ shows the results of the encapsulation efficiency (EE) of antioxidant compounds (QCT and TAA) from nanoliposomes prepared with low purity (Lp-SBL) and high purity (Hp-SBL) soybean lecithins. In the case of nanoliposomes loaded with QCT and TAA and prepared with Lp-SBL, the results of the encapsulation efficiency were around $88 \%$ and $57 \%$, respectively. For the nanoliposomes loaded with QCT and TAA and prepared with Hp-SBL, the results of the encapsulation efficiency were around $99 \%$ and $27 \%$, respectively. These results suggest that the Lp-SBL favored the encapsulation of polar compounds, while the Hp-SBL favored non-polar compound encapsulation. This result may be explained considering the differences between the lecithins' composition, where Lp-SBL displayed a higher amount of glycophospholipids and sugars, which could interact with polar compounds such as TAA through hydrogen bond interactions. Another study also suggested that some small molecules, such as caffeine, are mainly located in the solvation layer adjacent to the liposomal lipid bilayer interface [32]. On the contrary, the Hp-SBL exhibited a composition of phospholipids with a non-polar character and where the encapsulation of QCT (non-polar) could possibly take place by a process such as micellar solubilization.

On the other hand, Figure 4B shows the in vitro release of antioxidant compounds (QCT and TAA) from nanoliposomes prepared with low purity (Lp-SBL) and high purity (Hp-SBL) soybean lecithins. In the case of QCT, it is noteworthy that the release profiles display a lag time of $240 \mathrm{~min}$ for nanoliposomes made with Lp-SBL and a maximum released amount of $15 \%$. In comparison, for nanoliposomes elaborated with Hp-SBL, the lag time was $60 \mathrm{~min}$, and the maximum released amount was $40 \%$. These results are very interesting, since they show that the nanoliposomal vehicle considerably affects the QCT release, which can be easily appreciated when these are compared against the control (QCT alone, i.e., without a nanoliposomal vehicle), which is faster and exhibits a greater amount (99\%). This result is very consistent, considering that the QCT is encapsulated within the hydrophobic pseudo-phase formed by the lamellar structures of the hydrocarbon chains of phospholipids. Likewise, this result supports the previous results of the encapsulation efficiency, where the QCT is encapsulated in high amounts in both nanoliposomal systems (88-99\%), describing a greater affinity for the vehicle than the aqueous medium and therefore, its release is slow and controlled, as reflected in their respective lag times. On the contrary, the TAA-loaded nanoliposomes showed a faster release profile, regardless of the type of lecithin used. In this way, the TAA release from 
nanoliposomes described a similar behavior to that shown by the control (TAA alone), where rapid diffusion was observed. Regarding the control, it could be seen that around $80 \%$ of the TAA went through the bi-compartmental system in the first $5 \mathrm{~min}$, and then remained almost constant, suggesting that the material balance had been reached. In the case of the TAA loaded in the nanoliposomes, it was found that the release was lower $(\sim 60 \%)$, which indicates that most of the TAA is in the interfacial zone and not within the liposomal aqueous core and therefore, the release is practically immediate. Likewise, the difference in the amount of TAA between the control and the nanoliposomal systems suggests that there is a fraction of TAA inside the nanoliposome and to achieve a higher released amount, a longer time or other external conditions would possibly be required. Similar results have also been reported, where it has been described that, depending on the molecule polarity, the encapsulation and release mechanisms may vary [32-37].

(A) Antioxidant encapsulation

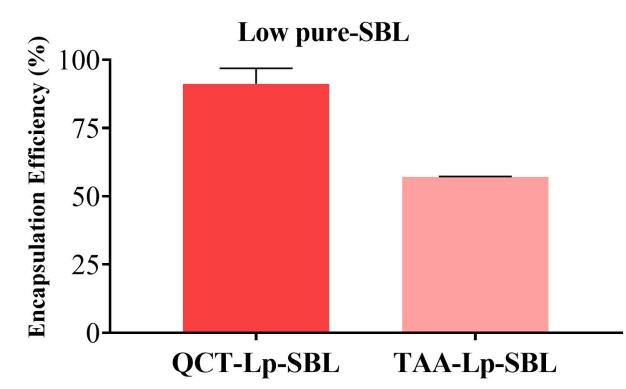

Hihg purity-SBL

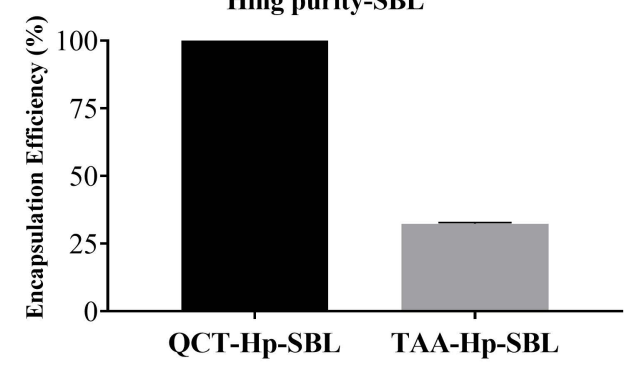

(B) Antioxidant release

TAA
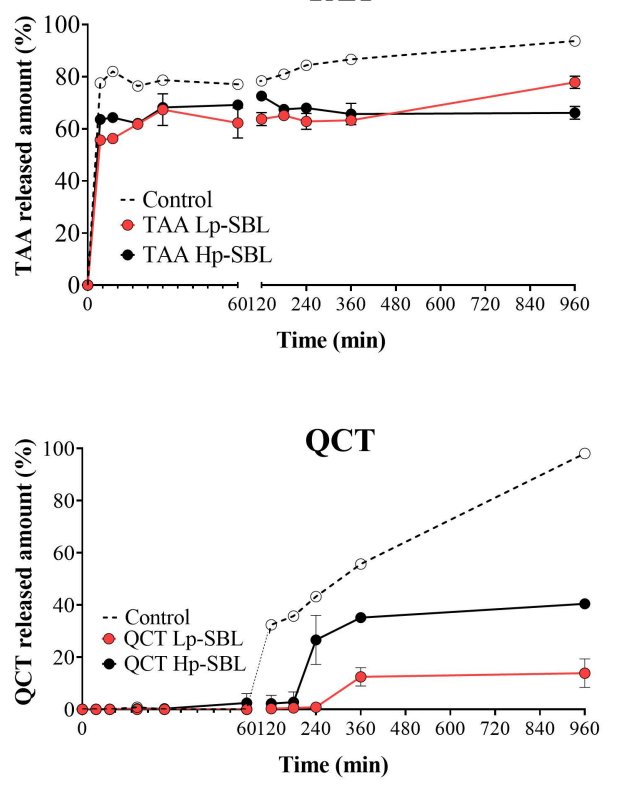

Figure 4. (A) Encapsulation efficiency and (B) release profiles of antioxidant (TAA and QCT) liposomes prepared with high purity lecithin (Hp-SBL) and low purity lecithin (Lp-SBL). QCT is quercetin and TAA is trans-aconitic acid. Data are reported as the mean $\pm \mathrm{SD}$ and $\mathrm{n}=3$.

\subsection{Antioxidant Effect Assay}

The ORAC assay results for pure QCT and TAA, as well as loaded in nanoliposomes, are shown in Figure 5. In the case of Trolox ${ }^{\circledR}$ (standard), QCT, and TAA antioxidants, the increase in their concentrations prevented fluorescence quenching of the probe in different ways. In the case of pure QCT, a similar trend to that described by the standard antioxidant was observed, while TAA exhibited a different behavior. The maximum antioxidant effect was reached at concentrations of 30.5, 40.5, and $140 \mu \mathrm{g} / \mathrm{mL}$ for Trolox ${ }^{\circledR}, \mathrm{QCT}$, and TAA, respectively (Figure 5A). These results can be explained by considering the photo-physical mechanism that takes place in the ORAC assay, as well as analyzing the chemical structures of antioxidants (Figure 5B). In this context, fluorescein in aqueous medium emits fluorescent radiation at $520 \mathrm{~nm}$, which remains practically unchanged over time. Then, the addition of the AAPH reagent leads to the oxidation of fluorescein by the reactive oxygen species (ROS) generated during the homolytic cleavage of such reagent [38,39]. Consequently, the oxidation in fluorescein leads to a decay of the fluorescence intensity over time (quenching) [38-40]. Therefore, the addition of an antioxidant establishes competition with fluorescein in the oxidation process and thus, when the 
antioxidant interacts with the ROS species, fluorescence decay is avoided, which is interpreted as an antioxidant effect. In these molecules, the phenyl substituent presents a thermodynamic equilibrium between phenyl and phenolate species, where the phenolate form is the one that reacts with the ROS species $[8,38,39,41,42]$. On the contrary, the antioxidant effect of TAA is due to the alteration of the phenyl-phenolate thermodynamic balance in fluorescein, leading to a slight predominance of the neutral form (R-phenyl), which is less reactive against ROS species. Moreover, the antioxidant effect was achieved at high concentrations $(140 \mu \mathrm{g} / \mathrm{mL})$, being moderate. However, it is important to note that TAA does not have oxidizable groups $[9,43]$, whereas the Trolox ${ }^{\circledR}$ and QCT antioxidants do and therefore, TAA's ability to avoid fluorescence decay is more limited.

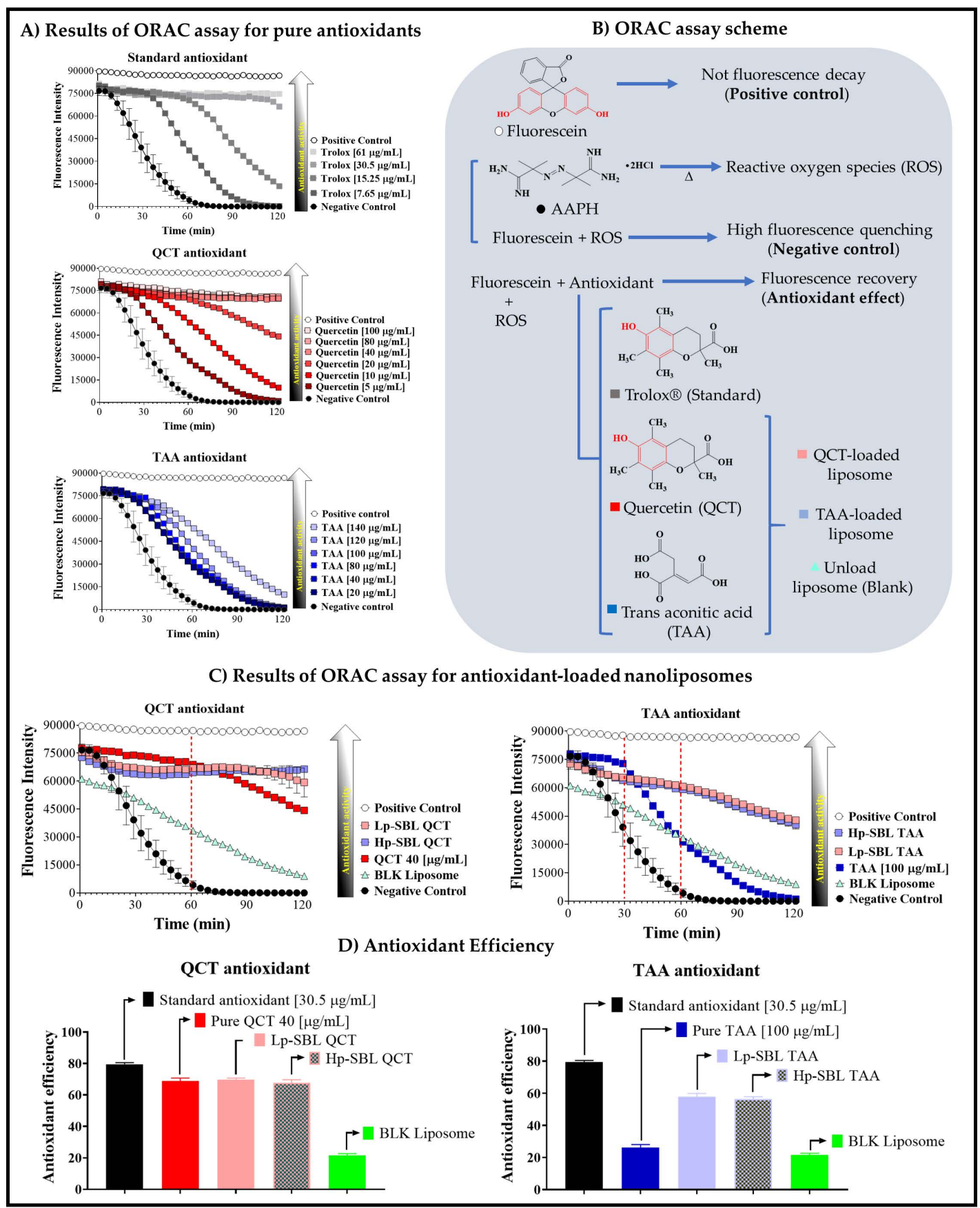

Figure 5. Results of the oxygen radical absorbance capacity (ORAC) assay. (A) Pure antioxidants. (B) ORAC method scheme. (C) Antioxidant-loaded nanoliposome. (D) Antioxidant efficiency. Data are reported as the mean $\pm \mathrm{SD}$ and $n=3$. 
Regarding nanoliposomes loaded with QCT and TAA (Figure 5C), the antioxidant effect changes, depending on the type of compound. In the case of pure QCT, slight fluorescence decay of the probe was observed after $30 \mathrm{~min}$, while QCT-loaded nanoliposomes displayed a sustained fluorescence effect. This result is consistent with that previously obtained in the QCT release profile from liposomes, where a sustained release was observed. Therefore, the use of nanoliposomes extends the antioxidant effect of QCT over time. On the contrary, TAA presented a considerable change when it was loaded in nanoliposomes, leading to a remarkable recovery of fluorescence. In the case of pure TAA, the antioxidant effect was very short and after $30 \mathrm{~min}$, the probe fluorescence was considerably quenched. However, when TAA was loaded on nanoliposomes, there was a slight recovery of the fluorescent emission, suggesting a slight increase in the antioxidant effect. However, this recovery was less than that obtained with Trolox ${ }^{\circledR}$ and QCT antioxidants. This result is consistent considering that TAA does not have the phenyl substituent, which is involved in the oxidation by ROS species.

Regarding the antioxidant efficiency (Figure 5D), the results showed that pure QCT and that loaded in the nanoliposomes have practically the same antioxidant efficiency of around $70 \%$, being very similar to that shown by the standard antioxidant. Nevertheless, the most interesting result is the sustained antioxidant effect over time, which was achieved when QCT was loaded inside the liposomes. In relation to TAA, the antioxidant efficiency was $<30 \%$, which is lower than the values for Trolox ${ }^{\circledR}$ and QCT antioxidants. However, this antioxidant efficiency was significantly improved when TAA was loaded in the nanoliposomes, resulting in an antioxidant efficiency value of around $60 \%$. Regarding the type of lecithins used for the formation of nanoliposomes, no significant changes were observed concerning the antioxidant effect. In the case of Lp-SBL-QCT and Hp-SBL-QCT systems, as well as Lp-SBL-TAA and Hp-SBL-TAA systems, the antioxidant efficiencies were very similar to each other, with values of around $70 \%$ and $60 \%$, respectively. Likewise, it was observed that both liposomal systems also exhibit a slight antioxidant effect of around $20 \%$. These results are very interesting because they show that nanoliposomes formed with low purity lecithins can provide a similar antioxidant effect to that provided with nanoliposomes formed with high purity lecithins.

\section{Material and Methods}

\subsection{Materials}

Low purity grade lecithin (Lp-SBL) was obtained from Farmacia-Drogueria San Jorge Ltd.a (Cali, Colombia), whereas high purity grade lecithin (Hp-SBL) (Epikuron 200 ${ }^{\mathrm{TM}}, \mathrm{Mw}=786 \mathrm{~g} / \mathrm{mol}$ ) was purchased from Cargill Corporation (Wayzata, MN, USA). Both lectins claim to come from the same plant source (soybean) and were used as received. The phospholipid 1,2-dioleoylsn-glycero-3-phosphoethanolamine (DOPE, $\mathrm{Mw}=744.03 \mathrm{~g} / \mathrm{mol}$ ) and cholesterol $(\mathrm{Mw}=386 \mathrm{~g} / \mathrm{mol}$ ) were purchased from Avanti Polar Lipids (Alabaster, AL, USA). Quercetin (QCT), trans-aconitic acid (TAA), and Ethanol USP grade were purchased from Sigma-Aldrich (St. Louis, MO, USA) and ultrapure water was supplied by an Elix Essential Millipore ${ }^{\circledR}$ purification system, with a mean conductivity value of $\sim 0.050 \mu \mathrm{Scm}$. Methanol Lichrosolv ${ }^{\mathrm{TM}}$ mass spectrometry grade was obtained from Sigma-Aldrich (St. Louis, MO, USA). Regarding the antioxidant activity assay, the analytical reagents employed were fluorescein sodium salt, 2,2'-Azobis(2-methylpropionamidine) dihydrochloride (AAPH), 6-Hydroxy-2,5,7,8-tetramethylchromane-2-carboxylic acid (Trolox), potassium phosphate monobasic, and potassium phosphate dibasic, which were purchased from Sigma-Aldrich (Merck KGaA, Darmstadt, Germany)

\subsection{Chemical Characterization of Lecithins}

\subsubsection{Infrared Spectroscopy (FTIR) Characterization}

The structural characterization of lecithins was performed in an FT-infrared spectrometer coupled to an attenuated total reflectance (ATR) instrument (Nicolet 6700, Thermo Fisher Scientific, 
Waltham, MA, USA). The spectra were recorded by using an attenuated total reflectance Smart iTR ${ }^{\mathrm{TM}}$ accessory, where the spectra of both lectins were compared.

\subsubsection{Thermal Analysis}

Thermal studies were carried out on a Q2000 differential scanning calorimeter (DSC; TA Instruments, New Castle, DE, USA) calibrated with indium $\mathrm{T}_{\mathrm{m}}=155.78^{\circ} \mathrm{C}$ and $\Delta \mathrm{H}_{\mathrm{m}}=28.71 \mathrm{~J} / \mathrm{g}$. Therefore, a modulated heating-cooling cycle from $-10{ }^{\circ} \mathrm{C}(263.15 \mathrm{~K})$ to $250{ }^{\circ} \mathrm{C}(523.15 \mathrm{~K})$ at a heating rate of $5{ }^{\circ} \mathrm{C} / \mathrm{min}$ was used. It was applied a $\pm 0.5^{\circ} \mathrm{C}$ modulation each $40 \mathrm{~s}$. Approximately $10 \mathrm{mg}$ of each sample was placed on a hermetic crucible with a lid, and an empty hermetic crucible was used as a reference.

\subsubsection{Electrospray Ionization Mass Spectrometry}

A methanolic solution of each respective lecithin was prepared at a concentration of $1 \mathrm{mg} / \mathrm{mL}$, which was injected by direct infusion (flow of $3 \mu \mathrm{L} / \mathrm{min}$ ) into a simple quadrupole mass spectrometer coupled to an electrospray ionization source (SQD2/ESI, Waters Corporation, Milford, MA, USA). The spectra were obtained in total ion scanning mode for positive ions $\left(\mathrm{ESI}^{+}\right)$and negative ions $\left(\mathrm{ESI}^{-}\right)$, in a range of 200 to $2000 \mathrm{~m} / \mathrm{z}$. The test conditions for both ionization modes were as follows: Desolvation gas flow, $550 \mathrm{~L} / \mathrm{h}$; desolvation temperature, $500{ }^{\circ} \mathrm{C}$; source temperature, $150{ }^{\circ} \mathrm{C}$; extraction voltage, $3 \mathrm{~V}$; cone voltage, $40 \mathrm{~V}$; and capillary voltage $2.69 \mathrm{kV}$. The samples were injected in triplicate.

\subsection{Development of Nanoliposomes}

\subsubsection{Preparation by the Ethanol Injection Method}

The nanoliposomes were prepared based on a sequential process defined in several steps, depending on the antioxidant [44]. In the case of QCT-loaded nanoliposomes, they were prepared as follows: (i) Dispersion of phospholipids in organic phase: Ethanolic solutions of lecithin $(1.3 \mathrm{mg} / \mathrm{mL})$, cholesterol $(0.64 \mathrm{mg} / \mathrm{mL})$, DOPE $(1.23 \mathrm{mg} / \mathrm{mL})$, and QCT $(350.8 \mu \mathrm{g} / \mathrm{mL})$ were prepared. From those solutions, volumes of $30,11.5,30$, and $28.5 \mu \mathrm{L}$ were taken, respectively; (ii) mixture with aqueous phase: $100 \mu \mathrm{L}$ of organic phase was slowly added to $100 \mu \mathrm{L}$ of ultrapure water, and the solution was then stirred for $1 \mathrm{~min}$ and left to rest for $10 \mathrm{~min}$; and (iii) Nanoliposome formation: The resulting mixture between the organic phase and aqueous media was diluted in $800 \mu \mathrm{L}$ of ultra-pure water. This process led to nanoliposome formation with a final QCT concentration of $35 \mu \mathrm{g} / \mathrm{mL}$. In the case of TTA-loaded nanoliposomes, these were prepared as follows: (i) Dispersion of phospholipids in organic phase: Ethanolic solutions of lecithin $(1.3 \mathrm{mg} / \mathrm{mL})$, cholesterol $(0.64 \mathrm{mg} / \mathrm{mL})$, and DOPE $(1.23 \mathrm{mg} / \mathrm{mL})$ were prepared. From those solutions, volumes of 42.4, 15.2, and $42.4 \mu \mathrm{L}$ were taken, respectively. (ii) Mixture with aqueous phase: $100 \mu \mathrm{L}$ of organic phase was slowly added to $100 \mu \mathrm{L}$ of aqueous media trans-aconitic acid solution with a $1000 \mu \mathrm{g} / \mathrm{mL}$ concentration (Ultra-pure water), and the sample was then stirred for $1 \mathrm{~min}$ and left to rest for $10 \mathrm{~min}$. The nanoliposome formation was conducted similarly to QCT-loaded nanoliposomes, where the TAA concentration in nanoliposomes was $100 \mu \mathrm{g} / \mathrm{mL}$. Liposomes were purified by means of the ultrafiltration/centrifugation technique. An aliquot of each nanoliposome dispersion was transferred into an ultrafiltration tube (VWR, modified polyethersulfone-PES $10 \mathrm{kDa}$, $500 \mu \mathrm{L}$, diameter: $0.96 \mathrm{~cm}$ ) and centrifuged (MIKRO 185, Hettich Lab Technology, Tuttlingen, Germany) at 10,000 rpm (1075 RFC) for $6 \mathrm{~min}$. Each nanoliposomal system was prepared in triplicate at room temperature $\left(25^{\circ} \mathrm{C}\right)$.

\subsubsection{Physicochemical Characterization}

The particle size and zeta potential were determined using a Zetasizer nano ZSP (Malvern Instrument, Worcestershire, UK) with a red $\mathrm{He} / \mathrm{Ne}$ laser $(633 \mathrm{~nm})$. The particle size was measured using dynamic light scattering (DLS) with an angle of scattering of $173^{\circ}$ at $25^{\circ} \mathrm{C}$, in a quartz flow cell (ZEN0023), whereas the zeta potential was measured using a disposable folded capillary cell 
(DTS1070). This instrument reports the particle size as the mean particle diameter (z-average), with the polydispersity index (PDI) ranging from 0 (monodisperse) to 1 (very broad distribution). All measurements were performed in triplicate after an appropriate dilution $(\sim 5: 5000, v / v)$ of the liposome suspension in ultra-pure water and are reported as the mean and standard deviation of measurements made from freshly prepared liposomal dispersions.

\subsection{Encapsulation and In Vitro Release of Antioxidant Compounds}

\subsubsection{Antioxidant Encapsulation Efficiency (EE)}

The EE of QCT and TAA was assessed using the ultrafiltration/centrifugation technique. An aliquot of each nanoliposome dispersion was transferred into an ultrafiltration tube (VWR, modified polyethersulfone-PES $10 \mathrm{kDa}, 500 \mu \mathrm{L}$, diameter: $0.96 \mathrm{~cm}$ ) and centrifuged (MIKRO 185, Hettich Lab Technology, Tuttlingen, Germany) at 10,000 rpm (1075 RFC) for $6 \mathrm{~min}$. For QCT quantification, an aliquot of the filtrate obtained in each system was obtained and evaluated with a microplate reader (Synergy, H1. Microplate reader, Biotek, Winooski, VT, USA). The amount of quercetin was determined by interpolation from a calibration curve that was previously prepared at the following concentrations, using ultra-pure water as the solvent: $1,3,10,30$, and $300 \mu \mathrm{g} / \mathrm{mL}$. For the quantification of TAA, an aliquot of the filtrate obtained in each system was obtained and evaluated via HPLC (Lachrom elite, Merck, Darmstadt, Germany) equipped with a photo diode array (PDA) detector and an automatic sampling system. The mobile phase consisted of acetonitrile and water with a $\mathrm{pH}$ of 2.5 (10:90), and the flow rate was $0.8 \mathrm{~mL} / \mathrm{min}$. Separation was achieved using a $50 \mathrm{~mm} \times 4.6 \mathrm{~mm}$, Zorbax Eclipse XDB-C18 (Agilent technologies, Santa Clara, CA, USA) reversed-phase column, with an average particle size of $1.8 \mu \mathrm{m}$, keeping the column at $25^{\circ} \mathrm{C}$. The column effluent was monitored at $270 \mathrm{~nm}$, and the chromatographic data analysis was performed with EZChrome software (Agilent technologies, Santa Clara, CA, USA). The amount of TAA was determined by interpolation from a calibration curve that was previously prepared at the following concentrations, using ultra-pure water as the solvent: 5, 10, 20, 40, 80, and $100 \mu \mathrm{g} / \mathrm{mL}$. Finally, the amount of QCT and TAA loaded inside the nanoliposomes was calculated using the following equation:

$$
E E=100-\left[\frac{Q_{t}-Q_{s}}{Q_{t}} \times 100\right]
$$

where $E E, Q_{t}$, and $Q s$ correspond to the encapsulation efficiency, initial total amount of bioactive compound, and amount of bioactive compound in the filtrate, respectively.

\subsubsection{In Vitro Antioxidant Release}

The in vitro release of QCT and TAA from nanoliposomes was assessed by the dialysis method, using $5 \mathrm{~mL}$ of phosphate buffer with a $\mathrm{pH}$ of 7.0 and $150 \mathrm{mM}$ aqueous medium under sink conditions. Therefore, volumes of $500 \mu \mathrm{L}$ of the nanoliposomes were placed into a dialysis tube (VWR, modified polyethersulfone-PES cut-off $10 \mathrm{kDa}, 500 \mu \mathrm{L}$, diameter: $0.96 \mathrm{~cm}$ ) in triplicate and dialyzed at $37^{\circ} \mathrm{C}$ for $16 \mathrm{~h}$ with constant stirring in an incubated orbital shaker (Inkubator 1000 with Unimax 1010, Heidolph Instruments, Schwalbach, Germany). Subsequently, the samples were taken from the external medium at intervals of $0,5,10,20,30,60,120,180,240,360$, and $996 \mathrm{~min}$. The quantification of QCT and TAA was performed as described in the encapsulation efficiency section.

\subsection{Antioxidant Effect Assay}

The antioxidant activity was determined by the oxygen radical absorbance capacity (ORAC) assay $[45,46]$, which is a method based on an evaluation of the ability of a compound to prevent the fluorescence quenching mediated by the AAPH reagent. For this, fluorescein (a fluorescent probe) and AAPH solutions were prepared in PBS (pH: 7.0) at concentrations of $0.02 \mathrm{mg} / \mathrm{mL}$ and $59.8 \mathrm{mg} / \mathrm{mL}$, respectively. In contrast, the antioxidant compounds were made in PBS (pH: 7.0) at concentrations of 
$7.65,15.25,30.5$, and $61 \mu \mathrm{g} / \mathrm{mL}$ for the standard antioxidant (Trolox ${ }^{\circledR}$ ); $5,10,20,40,80$, and $100 \mu \mathrm{g} / \mathrm{mL}$ for QCT; and 20, 40, 80, 100, 120, and $140 \mu \mathrm{g} / \mathrm{mL}$ for TAA. The evaluation of the fluorescent decay for fluorescein was conducted using a Synergy H1 microplate reader (Biotek, Winooski, VT, USA), where excitation and emission wavelengths of 485 and $520 \mathrm{~nm}$ were used, respectively, at $37^{\circ} \mathrm{C}$. Measurements were carried out in triplicate every $3 \mathrm{~min}$ for $2 \mathrm{~h}$, and the data obtained from the fluorescent vs. time curves are reported as the average antioxidant efficiency (AE) of the antioxidant compound. This parameter is defined as the area under the fluorescent decay curve (AUC) recorded at a particular time in relation to the rectangular area $(\mathrm{R})$ described by $100 \%$ of fluorescent emission of pure fluorescein (AUC of the positive control) at the same time. Therefore, the antioxidant efficiency can be calculated from:

$$
A E=\frac{A U C}{R} \times 100 \%
$$

Finally, the pure fluorescein solution was named as the positive control because fluorescence decay does not take place. On the contrary, the fluorescein + AAPH solution was labeled as the negative control, because AAPH forms reactive oxygen species (ROS) that lead to a high quenching of the fluorescent probe. Similarly, Trolox ${ }^{\circledR}$ and unloaded nanoliposomes were labeled as the standard antioxidant and blank, respectively.

\subsection{Statistical Analysis}

The data were tabulated and analyzed using Microsoft Excel and Graph Pad Prism, respectively. The homogeneity of variance in the data was analyzed using Bartlett's test. Statistical comparisons were conducted using a one-way ANOVA. The Bonferroni post-hoc test was used to determine significant differences between the two independent groups. A confidence level of $95 \%$ was adopted. Data are expressed as the mean \pm standard deviation.

\section{Conclusions}

In this study, it was established that low and high purity lecithins from a similar plant source (soybean) show differences and similarities, which can mean both advantages and disadvantages for their use as raw materials. First, the physical appearance is a determining factor for obtaining adequate handling and organoleptic characteristics at different stages of the product life cycle. Accordingly, the physical handling of a material such as low purity degree lecithin presents some difficulties compared to a material such as high purity degree lecithin. However, the physicochemical characterization of both materials using instrumental techniques such as FTIR, MS, and DSC, indicated that the chemical compositions of the two lecithins are very similar. Therefore, phospholipids such as phosphatidylcholine and phosphatidyl inositol were the main constituents of lecithins in this work. Regarding the ability to form nanoliposomal systems with adequate physicochemical characteristics (particle sizes $<300 \mathrm{~nm}$, PDI $<0.3 \mathrm{~nm}$, and zeta potential values of $\sim-30 \mathrm{mV}$ ), it was found that both lectins allow the preparation of these types of soft nanometric vehicles in a simple way. On the other hand, it was established that lecithins with a low purity grade consist of a greater amount of polar phospholipids, which tend to mainly encapsulate trans-aconitic acid (TAA). In contrast, lecithins with a high purity degree and which mainly consist of non-polar phospholipids tend to encapsulate a higher amount of quercetin (QCT). It was also established that the release of antioxidant compounds from nanoliposomal systems depends on their polarity and the way that they are encapsulated. In the case of the TAA, this is mainly located in the nanoliposome-water interface, where its release is very fast and around $60 \%$. On the contrary, QCT release is slow and occurs at smaller quantities (15-40\%), which is explained by the specific location of QCT within the lamellar structure of the nanoliposomes, where its affinity for such pseudo phase is great and therefore, its release is limited. It was demonstrated that low purity lecithins represent a viable alternative in terms of costs-benefits for obtaining innovative products for application to the food and cosmetic sectors. On the other hand, it was found that QCT presents a high antioxidant efficiency, which is sustained over time, describing a behavior very similar 
to the standard. On the contrary, it was found that TAA has a low antioxidant efficiency that can be increased when it is loaded with nanoliposomes. Finally, it was found that nanoliposomes formed with low purity lecithins can provide an antioxidant effect equal to that provided with nanoliposomes formed with high purity lecithins.

Author Contributions: M.J.A. and J.D.S. mainly performed the test of development and physical characterization of nanoliposomes. C.J.Y. is mainly responsible for the physicochemical characterization of lecithins, the encapsulation efficiency, the in vitro release assays, and the test of antioxidant effect evaluation. J.O.-G. is mainly responsible for validation and C.H.S. is mainly responsible for supervision, data analysis, validation, and final manuscript preparation. All authors have read and agreed to the published version of the manuscript.

Funding: This research was funded by the internal grant of Icesi University No. CA0413107.

Acknowledgments: The authors thank the Icesi University for the funding provided for the development of the study and Santiago de Cali University for supporting the APC.

Conflicts of Interest: The authors declare no conflict of interests.

$\begin{array}{ll}\text { Abbreviations } \\ \text { QCT } & \text { Quercetin } \\ \text { TAA } & \text { Trans-aconitic acid } \\ \text { Lp-SBL } & \text { low purity-soybean lecithin } \\ \text { Hp-SBL } & \text { low purity-soybean lecithin } \\ \text { PC } & \text { phosphatidylcholine } \\ \text { PE } & \text { phosphatidylethanolamines } \\ \text { PS } & \text { phosphatidylserines } \\ \text { PI } & \text { phosphatidylinositols } \\ \text { PG } & \text { phosphatidylglycerols } \\ \text { GPL } & \text { glyphospholipids } \\ \text { PA } & \text { phosphatidic acid }\end{array}$

\section{References}

1. List, G.R. Soybean Lecithin: Food, Industrial Uses, and Other Applications. In Polar Lipids: Biology, Chemistry, and Technology; ACOS PRESS: Urbana, IL, USA, 2015; ISBN 9781630670450.

2. Gunstone, F.D. Phospholipid Technology and Applications; Oily Press: Bridgwater, UK, 2008; ISBN 9780955251221.

3. Klang, V.; Valenta, C. Lecithin-based nanoemulsions. J. Drug Deliv. Sci. Technol. 2011, 21, 55-76. [CrossRef]

4. Mozafari, M.R.; Johnson, C.; Hatziantoniou, S.; Demetzos, C. Nanoliposomes and their applications in food nanotechnology. J. Liposome Res. 2008, 18, 309-327. [CrossRef] [PubMed]

5. Scholfield, C.R. Composition of soybean lecithin. J. Am. Oil Chem. Soc. 1981, 58, 889-892. [CrossRef]

6. Palacios, L.E.; Wang, T. Egg-yolk lipid fractionation and lecithin characterization. JAOCS J. Am. Oil Chem. Soc. 2005, 82, 571-578. [CrossRef]

7. Szuhaj, B.F. Lecithin production and utilization. J. Am. Oil Chem. Soc. 1983, 60, 306-309. [CrossRef]

8. Lesjak, M.; Beara, I.; Simin, N.; Pintać, D.; Majkić, T.; Bekvalac, K.; Orčić, D.; Mimica-Dukić, N. Antioxidant and anti-inflammatory activities of quercetin and its derivatives. J. Funct. Foods 2018, 40, 68-75. [CrossRef]

9. Piang-Siong, W.; De Caro, P.; Marvilliers, A.; Chasseray, X.; Payet, B.; Shum Cheong Sing, A.; Illien, B. Contribution of trans-aconitic acid to DPPHrad scavenging ability in different media. Food Chem. 2017, 214, 447-452. [CrossRef]

10. Fernandes, G.D.; Alberici, R.M.; Pereira, G.G.; Cabral, E.C.; Eberlin, M.N.; Barrera-Arellano, D. Direct characterization of commercial lecithins by easy ambient sonic-spray ionization mass spectrometry. Food Chem. 2012, 135, 1855-1860. [CrossRef]

11. Verdonck, E.; Schaap, K.; Thomas, L.C. A discussion of the principles and applications of Modulated Temperature DSC (MTDSC). Int. J. Pharm. 1999, 192, 3-20. [CrossRef]

12. McPhillips, H.; Craig, D.Q.M.; Royall, P.G.; Hill, V.L. Characterisation of the glass transition of HPMC using modulated temperature differential scanning calorimetry. Int. J. Pharm. 1999, 180, 83-90. [CrossRef] 
13. Linares, V.; Yarce, C.J.; Echeverri, J.D.; Galeano, E.; Salamanca, C.H. Relationship between degree of polymeric ionisation and hydrolytic degradation of Eudragit ${ }^{\circledR}$ E polymers under extreme acid conditions. Polymers 2019, 11, 1010. [CrossRef] [PubMed]

14. Sandoval, A.; Rodriguez, E.; Fernandez, A. Application of analysis by differential scanning calorimetry (DSC) for the characterization of the modifications of the starch. Rev. Fac. MINAS 2005, 72, 45-53.

15. Hatakeyama, T.; Iijima, M.; Hatakeyama, H. Role of bound water on structural change of water insoluble polysaccharides. Food Hydrocoll. 2016, 53, 62-68. [CrossRef]

16. Nakamur, K.; Minagaw, Y.; Hatakeyam, T.; Hatakeyama, H. DSC studies on bound water in carboxymethylcellulose-polylysine complexes. Thermochim. Acta 2004, 416, 135-140. [CrossRef]

17. Yarce, C.; Pineda, D.; Correa, C.; Salamanca, C. Relationship between Surface Properties and In Vitro Drug Release from a Compressed Matrix Containing an Amphiphilic Polymer Material. Pharmaceuticals 2016, 9, 34. [CrossRef]

18. Marianecci, C.; Petralito, S.; Rinaldi, F.; Hanieh, P.N.; Carafa, M. Some recent advances on liposomal and niosomal vesicular carriers. J. Drug Deliv. Sci. Technol. 2016, 32, 256-269. [CrossRef]

19. Rafiee, Z.; Barzegar, M.; Sahari, M.A.; Maherani, B. Nanoliposomal carriers for improvement the bioavailability of high-Valued phenolic compounds of pistachio green hull extract. Food Chem. 2017, 220, 115-122. [CrossRef]

20. Taylor, T.M.; Davidson, P.M.; Bruce, B.D.; Weiss, J. Liposomal nanocapsules in food science and agriculture. Crit. Rev. Food Sci. Nutr. 2005, 45, 587-605. [CrossRef]

21. Hsieh, Y.F.; Chen, T.L.; Wang, Y.T.; Chang, J.H.; Chang, H.M. Properties of liposomes prepared with various lipids. J. Food Sci. 2002, 67, 2808-2813. [CrossRef]

22. Raut, S.; Bhadoriya, S.S.; Uplanchiwar, V.; Mishra, V.; Gahane, A.; Jain, S.K. Lecithin organogel: A unique micellar system for the delivery of bioactive agents in the treatment of skin aging. Acta Pharm. Sin. B 2012, 2, 8-15. [CrossRef]

23. Hasanovic, A.; Hollick, C.; Fischinger, K.; Valenta, C. Improvement in physicochemical parameters of DPPC liposomes and increase in skin permeation of aciclovir and minoxidil by the addition of cationic polymers. Eur. J. Pharm. Biopharm. 2010, 75, 148-153. [CrossRef] [PubMed]

24. Refai, H.; Hassan, D.; Abdelmonem, R. Development and characterization of polymer-coated liposomes for vaginal delivery of sildenafil citrate. Drug Deliv. 2017, 24, 278-288. [CrossRef] [PubMed]

25. Elnaggar, Y.S.R.; El-Refaie, W.M.; El-Massik, M.A.; Abdallah, O.Y. Lecithin-based nanostructured gels for skin delivery: An update on state of art and recent applications. J. Control. Release 2014, 180, 10-24. [CrossRef] [PubMed]

26. Zhao, L.; Temelli, F.; Chen, L. Encapsulation of anthocyanin in liposomes using supercritical carbon dioxide: Effects of anthocyanin and sterol concentrations. J. Funct. Foods 2017, 34, 159-167. [CrossRef]

27. Laouini, A.; Jaafar-Maalej, C.; Limayem-Blouza, I.; Sfar, S.; Charcosset, C.; Fessi, H. Preparation, Characterization and Applications of Liposomes: State of the Art. J. Colloid Sci. Biotechnol. 2012, 1, 147-168. [CrossRef]

28. Marinova, K.G.; Alargova, R.G.; Denkov, N.D.; Velev, O.D.; Petsev, D.N.; Ivanov, I.B.; Borwankar, R.P. Charging of Oil-Water Interfaces Due to Spontaneous Adsorption of Hydroxyl Ions. Langmuir 1996, 12, 2045-2051. [CrossRef]

29. Barea, M.J.; Jenkins, M.J.; Gaber, M.H.; Bridson, R.H. Evaluation of liposomes coated with a pH responsive polymer. Int. J. Pharm. 2010, 402, 89-94. [CrossRef]

30. Sabín, J.; Prieto, G.; Ruso, J.M.; Hidalgo-Álvarez, R.; Sarmiento, F. Size and stability of liposomes: A possible role of hydration and osmotic forces. Eur. Phys. J. E 2006, 20, 401-408. [CrossRef]

31. Cantor, S.; Vargas, L.; Rojas, O.E.A.; Yarce, C.J.; Salamanca, C.H.; Oñate-Garzón, J. Evaluation of the antimicrobial activity of cationic peptides loaded in surface-modified nanoliposomes against foodborne bacteria. Int. J. Mol. Sci. 2019, 20, 680. [CrossRef]

32. Budai, L.; Kaszás, N.; Gróf, P.; Lenti, K.; Maghami, K.; Antal, I.; Klebovich, I.; Petrikovics, I.; Budai, M. Liposomes for topical use: A physico-chemical comparison of vesicles prepared from egg or soy lecithin. Sci. Pharm. 2013, 81, 1151-1166. [CrossRef]

33. Herman, A.; Herman, A.P. Caffeine's mechanisms of action and its cosmetic use. Skin Pharmacol. Physiol. 2012, 26, 8-14. [CrossRef] 
34. Scherer, R.; Godoy, H.T. Antioxidant activity index (AAI) by the 2,2-diphenyl-1-picrylhydrazyl method. Food Chem. 2009, 112, 654-658. [CrossRef]

35. Li, M.; Du, C.; Guo, N.; Teng, Y.; Meng, X.; Sun, H.; Li, S.; Yu, P.; Galons, H. Composition design and medical application of liposomes. Eur. J. Med. Chem. 2019, 164, 640-653. [CrossRef] [PubMed]

36. Lombardo, D.; Kiselev, M.A.; Caccamo, M.T. Smart Nanoparticles for Drug Delivery Application: Development of Versatile Nanocarrier Platforms in Biotechnology and Nanomedicine. J. Nanomater. 2019. [CrossRef]

37. Yadav, A.V.; Murthy, M.S.; Shete, A.S.; Sakhare, S. Stability aspects of liposomes. Indian J. Pharm. Educ. Res. 2011, 45, 402-413.

38. Kuti, J.O; Konuru, H.B. Antioxidant Capacity and Phenolic Content in Leaf Extracts of Tree Spinach (Cnidoscolus spp.). J. Agric. Food Chem. 2004, 52, 117-121. [CrossRef]

39. Ehlenfeldt, M.K.; Prior, R.L. Oxygen radical absorbance capacity (ORAC) and phenolic and anthocyanin concentrations in fruit and leaf tissues of highbush blueberry. J. Agric. Food Chem. 2001, 49, 2222-2227. [CrossRef]

40. Güçlü, K.; Kibrisliogilu, G.; Özyürek, M.; Apak, R. Development of a fluorescent probe for measurement of peroxyl radical scavenging activity in biological samples. J. Agric. Food Chem. 2014, 62, 1839-1845. [CrossRef]

41. Huang, D.; Boxin, O.U.; Prior, R.L. The chemistry behind antioxidant capacity assays. J. Agric. Food Chem. 2005, 53, 1841-1856. [CrossRef]

42. Prior, R.L. Oxygen radical absorbance capacity (ORAC): New horizons in relating dietary antioxidants/bioactives and health benefits. J. Funct. Foods 2015, 18, 797-810. [CrossRef]

43. Montoya, G.; Londono, J.; Cortes, P.; Izquierdo, O. Quantitation of trans-aconitic acid in different stages of the sugar-manufacturing process. J. Agric. Food Chem. 2014, 62, 8314-8318. [CrossRef] [PubMed]

44. Arévalo, L.M.; Yarce, C.J.; Oñate-Garzón, J.; Salamanca, C.H. Decrease of antimicrobial resistance through polyelectrolyte-coated nanoliposomes loaded with $\beta$-lactam drug. Pharmaceuticals 2019, 12, 1. [CrossRef] [PubMed]

45. Zheng, W.; Wang, S.Y. Antioxidant activity and phenolic compounds in selected herbs. J. Agric. Food Chem. 2001, 49, 5165-5170. [CrossRef] [PubMed]

46. Barros, R.G.C.; Andrade, J.K.S.; Denadai, M.; Nunes, M.L.; Narain, N. Evaluation of bioactive compounds potential and antioxidant activity in some Brazilian exotic fruit residues. Food Res. Int. 2017, 102, 84-92. [CrossRef] [PubMed]

Sample Availability: Samples of the compounds are available from the authors.

Publisher's Note: MDPI stays neutral with regard to jurisdictional claims in published maps and institutional affiliations.

(C) 2020 by the authors. Licensee MDPI, Basel, Switzerland. This article is an open access article distributed under the terms and conditions of the Creative Commons Attribution (CC BY) license (http://creativecommons.org/licenses/by/4.0/). 